\section{JTI}

JOURNAL OF

TRAUMA AND INJURY

Received: July 10, 2019

Revised: August 27, 2019

Accepted: September 10, 2019

\section{Correspondence to}

Youngmo Cho, M.D.

Department of Emergency Medicine and Biomedical Research Institute, Pusan National University Hospital, Pusan $\mathrm{Na}$ tional University School of Medicine, 179 Gudeok-ro, Seo-gu, Busan 49241, Korea

Tel: +82-51-240-7503

Fax: +82-51-253-6472

E-mail: specialkey7@naver.com

\title{
Factors and Their Correlation with Injury Severity of Elderly Pedestrian Traffic Accidents
}

\author{
Tae gyu Hyun, M.D. ${ }^{1}$, Seok-Ran Yeom, M.D., Ph.D. ${ }^{1}$, Sung-Wook Park, M.D. ${ }^{1}$, \\ Deasup Lee, M.D. ${ }^{2}$, Hyung bin Kim, M.D. ${ }^{1}$, Il Jae Wang, M.D., Ph.D. ${ }^{1}$, \\ Byung Gwan Bae, M.D. ${ }^{1}$, Min keun Song ${ }^{1}$, Youngmo Cho, M.D. ${ }^{1}$ \\ ${ }^{1}$ Department of Emergency Medicine and Biomedical Research Institute, Pusan National \\ University Hospital, Busan, Korea \\ ${ }^{2}$ Department of Emergency Medicine, Pusan National University Yangsan Hospital, \\ Yangsan, Korea
}

Purpose: No previous study has assessed elderly pedestrian traffic accidents based on a nationwide database. This study aimed to help primary physicians who examine patients in emergency departments to determine and make prompt and accurate treatment decisions.

Methods: This study used data from the Emergency Department-based Injury Indepth Surveillance from 2013 to 2017, managed by the Korea Centers for Disease Control and Prevention. Pedestrians aged $\geq 65$ years were included, and using multivariate logistic regression multiple factors were analyzed to determine their relationship with injury severity.

Results: Of 227,695 subjects, 6,498 were included, of whom 2,065 (31.8\%) were severely injured. There were more female than male patients in all severity groups. Most accidents occurred in the afternoon and on general roads. In the multivariate analysis, the odds ratio (OR) of injury severity for male pedestrians was 1.165 (95\% confidence interval: 1.034-1.313, $p=0.012$ ). Older age of patients and the use of ambulances were associated with greater injury severity. The accident time affected the degree of injury severity; i.e., compared to dawn, injury severity increased in the morning (OR: 1.246, $p=0.047$ ) and decreased at night (OR: 0.678, $p<0.001$ ). A significant difference was noted in the correlation between the type of vehicle causing the accident and the accident severity; i.e., motorcycle accidents had lower severity than bicycle accidents (OR: $0.582, p=0.047$ ).

Conclusions: Injury severity was correlated with sex, age, transportation to the ED, TA onset time, and type of vehicle. The study results suggest that injury severity may be positively reflected in initial assessments and overall integrated treatments by physicians and in the related policies.

Keywords: Elderly; Pedestrians; Injury severity 


\section{INTRODUCTION}

Worldwide, the aging society comprises $>7 \%$ of the population aged $>65$ years [1]. South Korea was known to have an aging society in 2000, with $>14 \%$ accounting for the aged society in 2018. Furthermore, $>20 \%$ of the population is known as the super-aged society, and according to the population growth trend, it is expected to exceed $20 \%$ by $2026[2,3]$. Older adults have poor health, vision, hearing, and reaction speed [4]. Moreover, the perceptual and cognitive problems of many older adults include reduced ability to maintain general attention, difficulty in categorizing non-critical information from important information, reduced speed and accuracy of processing information, reduced ability in solving problems, and reduced short-term memory [5]. The physical aging of these elderly people makes them vulnerable to exposure to diseases, and they are more likely to have a greater degree of impairment, assuming that traumatic injury is diagnostically the same as that in younger people.

According to the nation's Traffic Accident Analysis System, the number of pedestrian accidents has been declining, from 51,145 in 2014 to 45,921 in 2018. However, the number of accidents involving senior citizens has increased, from 10,828 in 2014 to 11,815 in 2018, which is equivalent to an increase from $21.2 \%$ to $25.7 \%$. According to the number of pedestrian mortalities, the mortality rate increased from $48.1 \%(919 / 1,910$ people aged $>65$ years) in 2014 to $56.6 \%$ (842/1,487 people aged $>65$ years) in 2018 [6]. According to a study in Victoria, Australia, people aged $>65$ years accounted for $14.6 \%$ of the total population but $39 \%$ of pedestrian mortalities. Based on further categorization, people aged 65-74 years accounted for $8 \%$ of the total population but $11 \%$ of pedestrian mortalities, whereas people aged between 75 and 84 years accounted for $5 \%$ of the total population but $14 \%$ of pedestrian mortalities [7]. As mentioned above, the national government recognized that the mortality rate of elderly pedestrian traffic accidents was high, which was reflected in the design and construction of actual roads and improvements of its policy. However, no previous study assessed pedestrian traffic accidents based on the national database in South Korea. Therefore, using the national database of South Korea, this study aimed to identify factors that affected the severity of traffic accidents of elderly pedestrians to help primary physicians treating such patients in emergency departments (EDs) predict injury severity as well as determine and provide prompt and accurate treatments.

\section{METHODS}

\section{Data collection and patient enrollment}

This study used data from the Emergency Department-based Injury In-depth Surveillance (EDIIS), which has been managed by the Korea Centers for Disease Control and Prevention (KCDC) since 2006. EDIIS is a collection of data on all injured patients who visit EDs that participate in a surveillance system to develop national policies for injury prevention. All types of injury data were collected by trained coordinators at each hospital and were entered into the KCDC online system. KCDC periodically performs quality control by analyzing errors. The number of EDs that participated in the data collection has increased from 5 in 2006 to 21 in 2010, and 23 in 2015 [8].

Data from January 2013 to December 2017 were obtained. Since 2013, the number of participating hospitals has increased to 23 , and data collection has been stable. Among the patients visiting EDs because of pedestrian traffic accidents, as defined by the Road Traffic Act in South Korea, patients aged $\geq 65$ years were included in the study. Except for injuries caused by rail vehicles, injuries related to all other types of vehicles, from bicycle to cultivators, were included. Patients missing any essential information related to Excess Mortality Ratio-adjusted Injury Severity Score (EMR-ISS) were excluded.

\section{Variable definitions}

The baseline characteristics of the patients included sex, age, transportation to ED, use of alcohol, time of arrival, types of vehicle, type of road, disposition at ED, and mortality based on data extracted from EDIIS. Transportation to ED was divided into whether or not an ambulance was used, including those using private ambulances and aircraft. When alcohol intake was suspected, it was noted. However, if alcohol consumption was not known, it was 
classified as unknown. The time of arrival was divided into four categories: dawn (00:00-05:59), morning (06:0011:59), afternoon (12:00-17:59), and night (18:00-23:59). For the type of vehicle, the speed and mass that affected the law of force and the appearance characteristics were considered, and the type of vehicle was subdivided into five types according to the classification in EDIIS: bicycles, motorcycles, general vehicles (four-wheel vehicles such as minibuses with $<20$ seats), large-sized vehicles (including all vehicles larger than small vehicles), and cultivator. The type of road was classified into three categories, namely alley/farm road, general road, and highway, and reflected all expected road characteristics and vehicle type and speed. However, if information was not acquired by the researcher, it was classified as unknown. The disposition at ED was divided into discharge, transfer, admission, and expired, and mortality was divided into survival and mortality. The EMR-ISS was based on the International Classification of Diseases 10th Edition, a method developed to measure injury severity of large-scale data developed in 2009 [9]. The EMR-ISS assigned patients scoring 1-24, indicating mild and moderate injuries, to the non-severe group and those scoring $\geq 24$, indicating severe and critical injuries, to the severe group $[10,11]$. The injury severity was determined using EMR-ISS instead of the days of hospitalization or mortality, which reflected the difference in physical status between the morbidity and mortality of elderly and younger patients with the same injury to ensure the validity of the patient's severity.

\section{Statistical analysis}

Categorical data were expressed in numbers and percentages, whereas numerical data were expressed as medians and interquartile ranges. The chi-square test and Mann-Whitney $U$-test were used to compare the differences in the characteristics of the severely and non-severely injured elderly pedestrian groups. To identify risk factors related to injury severity of elderly pedestrians, a stepwise backward multivariate logistic regression analysis was performed. Adjusted odds ratio (OR) with 95\% confidence interval was calculated. Data analyses were performed using SPSS version 22.0 (IBM Corp., Armonk, NY, USA), and $p$ values $<0.05$ were considered significant.

\section{Ethics statement}

This study was approved by the KCDC and an institutional review board (IRB) (IRB number H-1906-010-080). Because the data were analyzed anonymously, the need for informed consent was waived by the IRB.

\section{RESULTS}

Overall, 227,695 traffic accidents occurred during the study period, and 9,657 pedestrians were aged $\geq 65$ years. After excluding patients with missing essential information on factors affecting injury severity, 6,498 patients were included in the analysis (Fig. 1).

The proportion of female patients in both the severe and non-severe groups was higher than that of male patients, regardless of the injury severity. There were 1,228 (59.5\%) and 2,803 (63.2\%) female patients in the severe and non-severe group, respectively, and there was a significant difference ( $p=0.004)$ (Table 1). All other factors such as age, transportation to, use of alcohol, time of arrival, types of vehicle, type of road, disposition at $\mathrm{ED}$, and mortality $(p<0.001)$ were also statistically significant. In particular, patients in the severe group were older, used the ambulance more, and ingested alcohol more frequently than those in the non-severe group. The severe and non-severe groups had the lowest accident rates at dawn, i.e., 10.8\%

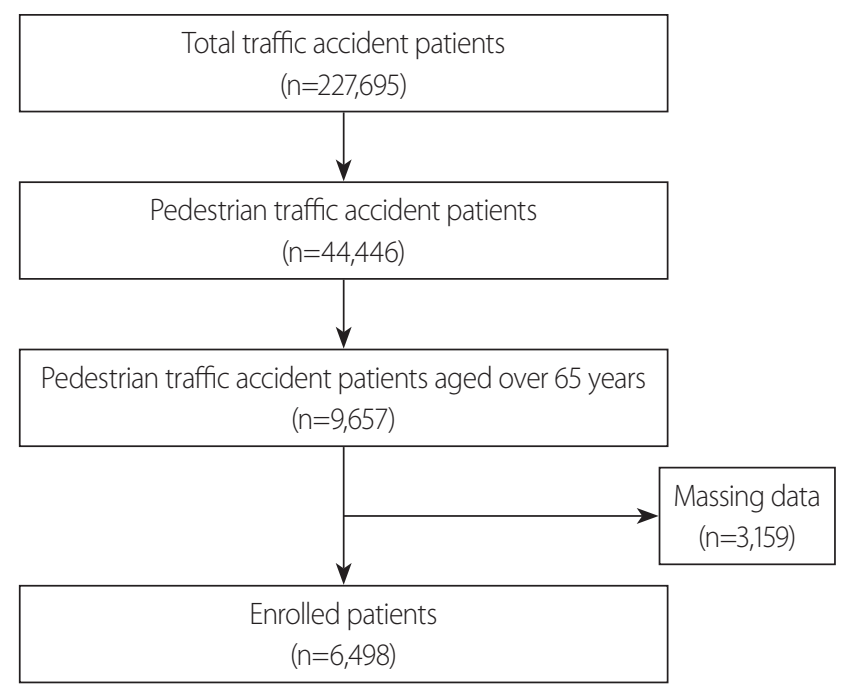

Fig. 1. Flow chart of study patients. 
Table 1. Baseline characteristics of the elderly pedestrian traffic accident

\begin{tabular}{|c|c|c|c|}
\hline Characteristic & $\begin{array}{l}\text { Severe group } \\
\quad(n=2,065)\end{array}$ & $\begin{array}{c}\text { Non-severe } \\
\text { group }(n=4,433)\end{array}$ & $p$-value \\
\hline Sex & & & 0.004 \\
\hline Female & $1,228(59.5)$ & $2,803(63.2)$ & \\
\hline Age (years) & 74 (70-79) & $73(69-78)$ & $<0.001$ \\
\hline Transportation to ED & & & $<0.001$ \\
\hline Ambulance & $1,921(93.0)$ & $2,837(64.0)$ & \\
\hline Others & $144(7.0)$ & 1,596 (36.0) & \\
\hline Use of alcohol & & & $<0.001$ \\
\hline Yes & $99(4.8)$ & $144(3.2)$ & \\
\hline No & $1,695(82.1)$ & $3,920(88.4)$ & \\
\hline Unknown & $271(13.1)$ & $369(8.3)$ & \\
\hline TA onset time (hour) & & & $<0.001$ \\
\hline 00:00-05:59 & $222(10.8)$ & $304(6.9)$ & \\
\hline 06:00-11:59 & $678(32.8)$ & 1,357 (30.6) & \\
\hline 12:00-17:59 & $602(29.2)$ & 1,712 (38.6) & \\
\hline 18:00-23:59 & $563(27.3)$ & $1,060(23.9)$ & \\
\hline Types of vehicle & & & $<0.001$ \\
\hline Bicycle & $47(2.3)$ & $240(5.4)$ & \\
\hline Motorcycle & $88(4.3)$ & $215(4.8)$ & \\
\hline General vehicle & $1,619(78.4)$ & $3,488(78.7)$ & \\
\hline Large-sized vehicle & $277(13.4)$ & $402(9.1)$ & \\
\hline Cultivator & $34(1.6)$ & $88(2.0)$ & \\
\hline Type of road & & & $<0.001$ \\
\hline Highway & $8(0.4)$ & $5(0.1)$ & \\
\hline General road & 1,823 (88.3) & $3,287(74.1)$ & \\
\hline Alley/farm road & 207 (10.0) & $1,094(24.7)$ & \\
\hline Unknown & $27(1.3)$ & $47(1.1)$ & \\
\hline Disposition at ED & & & $<0.001$ \\
\hline Discharge & $169(8.2)$ & $2,673(60.3)$ & \\
\hline Transfer & $236(11.4)$ & $400(9.0)$ & \\
\hline Admission & $1,410(68.3)$ & $1,259(28.4)$ & \\
\hline Expired & $250(12.1)$ & $101(2.3)$ & \\
\hline Mortality & & & $<0.001$ \\
\hline Survival & $1,595(77.2)$ & 4,292 (96.8) & \\
\hline Death & $470(22.8)$ & $141(3.2)$ & \\
\hline
\end{tabular}

Values are presented as mean (range) or number (\%). ED: emergency department, TA: traffic accident. and $6.9 \%$, respectively. When integrating the number of accidents between the two groups, the most frequent accident time was afternoon. However, the severe group had the highest frequency of accidents in the morning (32.8\%), whereas the non-severe group had the highest frequency of accidents at night (38.6\%). The type of vehicle causing the most accidents was a general vehicle in both the severe and non-severe groups, at $78.4 \%$ and $78.7 \%$, respectively. Similarly, both groups experienced the highest number of accidents on the general road ( $88.3 \%$ vs. $74.1 \%)$. The results of the disposition at ED revealed that admission was the highest in the severe group (68.3\%) and discharge was the highest in the non-severe group $(60.3 \%)$. In addition, the mortality rate was higher in the severe group than in the non-severe group (12.1\% vs. $2.3 \%)$. Overall mortality, including outcomes in ED, was also higher in the severe group than in the non-severe group (22.8\% vs. $3.2 \%)$.

Multivariate logistic regression analysis was performed with variables such as sex, age, transportation to $\mathrm{ED}$, use of alcohol, time of arrival, types of vehicle, and types of road to identify risk factors associated with EMR-ISS (Table 2). The analysis revealed that the OR of injury severity for male pedestrian patients was 1.165 (95\% confidence interval: $1.034-1.313, p=0.012$ ). In addition, the age of patients and the use of ambulance had higher ORs of severe injury $(p<0.001)$. Furthermore, the accident time affected the injury severity; i.e., compared with the severity at dawn, the degree of severity increased in the morning (OR: 1.246, $p=0.047$ ); in contrast, it decreased at night (OR: $0.678, p<0.001)$. Regarding the type of vehicle causing the accident, the injury severity in motorcycle accidents decreased compared with that in bicycle accidents (OR: $0.582, p=0.047$ ), although other types of vehicles were not associated with increased risk of mortality (Table 2).

\section{DISCUSSION}

This study aimed to identify factors that affected injury severity of elderly pedestrians involved in traffic accidents. Based on baseline characteristics, the female-tomale ratio was high in both the severe and non-severe groups. However, multivariate logistic regression analysis revealed that male and not female pedestrians were at a 
Table 2. Risk factors for injury severity in elderly pedestrian traffic accident patients

\begin{tabular}{|c|c|c|c|}
\hline \multirow{2}{*}{ Variable } & \multicolumn{2}{|c|}{ Adjusted } & \multirow{2}{*}{$p$-value } \\
\hline & OR & $95 \% \mathrm{Cl}$ & \\
\hline \multicolumn{4}{|l|}{ Sex } \\
\hline Female & 1 & & \\
\hline Male & 1.165 & $1.034-1.313$ & 0.012 \\
\hline Age (years) & 1.031 & $1.022-1.040$ & $<0.001$ \\
\hline \multicolumn{4}{|l|}{ Transportation to ED } \\
\hline Ambulance & 1 & & \\
\hline Others & 0.736 & $0.529-1.023$ & $<0.001$ \\
\hline \multicolumn{4}{|l|}{ Use of alcohol } \\
\hline Yes & 1 & & \\
\hline No & 0.736 & $0.529-1.023$ & 0.068 \\
\hline \multicolumn{4}{|l|}{ TA onset time (hour) } \\
\hline 00:00-05:59 & 1 & & \\
\hline 06:00-11:59 & 1.246 & $1.003-1.548$ & 0.047 \\
\hline 12:00-17:59 & 0.872 & $0.750-1.014$ & 0.076 \\
\hline 18:00-23:59 & 0.678 & $0.583-0.789$ & $<0.001$ \\
\hline \multicolumn{4}{|l|}{ Types of vehicle } \\
\hline Bicycle & 1 & & \\
\hline Motorcycle & 0.582 & $0.340-0.994$ & 0.047 \\
\hline General vehicle & 0.888 & $0.540-1.462$ & 0.641 \\
\hline Large-sized vehicle & 1.162 & $0.758-1.782$ & 0.491 \\
\hline Cultivator & 1.443 & $0.917-2.271$ & 0.113 \\
\hline \multicolumn{4}{|l|}{ Type of road } \\
\hline Highway & 1 & & \\
\hline General road & 1.663 & $0.476-5.814$ & 0.426 \\
\hline Alley/farm road & 0.719 & $0.426-1.213$ & 0.217 \\
\hline
\end{tabular}

OR: odds ratio, Cl: confidence interval, ED: emergency department, TA: traffic accident

higher risk of severe injury due to a traffic accident. Two previous studies reported similar results, suggesting the underlying causes for traffic accident-related mortality among the older people might be the same. For example, in a London-based study, the number of female pedestrian patients was higher than the number of male patients because of differences in cognition, strength, physical mobility and fitness, and road behavior. At the same time, Wouter's Newcastle results reported higher mortality and severity in male patients [12]. According to an Australian study, people's walking speed decreases with age, and women tend to walk more slowly than men. This slowness might be caused by aging muscles that weaken the strength of the legs; however, several training programs can improve muscle strength, balance, and walking speed [7].

The OR of the correlation between aging and severity was 0.031 , which was statistically significant $(p<0.001)$. Although this finding might have been a predictable outcome, the following evidence further strengthens it. First, for elderly pedestrians, the incidence of physical and mental deterioration may increase because of various physical and mental constraints that decrease their response capacity [13]. Additionally, the study that explained the association between cognitive abilities and traffic accidents revealed that patients aged $\geq 70$ years had lower cognitive scores and higher traffic accident rates than those aged 65-69 years [14]. Finally, a study from the UK reported that the number of pedestrian accidents has been decreasing, indicating that people's mobility has changed from walking to vehicles. However, senior citizens aged $\geq 65$ years (especially those aged $\geq 80$ years) still reported their walking distance, thereby increasing the rate of elderly pedestrian accidents and mortality [12].

When we investigated the correlation between alcohol consumption and injury severity, we found a high rate of drinking in the severe group, although it was not statistically different in the multivariate analysis. However, studies conducted in North Carolina have shown drinking as a major factor associated with pedestrian mortalities for all ages, especially for elderly pedestrians because alcohol affects judgment and reaction time, and even a relatively small amount of alcohol might have an exaggerated effect, especially when ingested along with prescription drugs often taken by older people [15]. Conversely, considering that elderly pedestrians consume less alcohol than younger patients, a previous study reported that alcohol was no correlated with the severity of elderly pedestrian traffic accidents [7]. Studies have shown that alcohol consumption ranges from $9 \%$ to $15 \%$ in the severely injured population during elderly pedestrian traffic accidents $[16,17]$, and our data also showed very low rates of alcohol consumption among elderly patients (3.7\%). Therefore, our results that there is no significant relationship between 
injury severity and alcohol are likely to be valid. However, there are some limitations to alcohol-related data. In South Korea, because the blood alcohol test is not routinely performed in EDs, the data regarding alcohol consumption are entirely dependent on the statement of the patient or their guardians.

The accident time that affected injury severity was morning (06:00-11:59) (OR: 1.246, $p=0.047)$, and the severity significantly decreased at night (18:00-23:59) (OR: $0.678, p<0.001)$ in the multivariate analysis. As in this study, a previous study revealed that the elderly people have a higher incidence of daytime accidents and a higher degree of severity owing to differences in life styles and walking patterns compared with people in the other age groups. Light conditions are generally an important part of time factor; however, for elderly pedestrians, a time zone that shows robust activity was the most important [15].

The multivariate analysis of the type of vehicle and road showed no relationship, except motorcycle severity was significantly lower (OR: $0.582, p=0.047$ ). The formula for determining mass and speed is force, which is expected to increase in severity because the vehicle is larger; however, no statistical significance was noted, and thus, further studies are required. As with the study published by Knoblauch and Crigler [18], the most accident-causing vehicles in our baseline theory were the general vehicles, and the most frequent accidents occurred on general roads. The highest frequency of accidents because of general vehicles and roads was thought to be preventable by improving overall traffic policies and road environments. Unlike regulations on vehicles and facilities, such policies and road environments cannot be strict with regard to pedestrians; thus, the cause of accidents can be more responsible for pedestrians. Pedestrian accidents also occur because of unsafe pedestrian behavior, and elderly people are among the most common violators [18]. Pedestrian training is needed, and the Walk Alert Pedestrian Safety Program developed by the National Safety Council and the U.S. Department of Transportation has proposed items for pedestrians to be cautious about such as ensuring proper search behavior and being seen; checking traffic signals, signs, and markings; marking a disabled person's vehicle, as suitable; crossing at intersections; and using visual screens [19].

This study had some limitations. It was a cross-sectional study involving nationwide data involving 23 hospitals nationwide and not a population-based study. Considering the characteristics of data collection organizations, consisting of 10 level-1 emergency centers and 12 level-2 emergency centers from the three categories of EDs classified by the Ministry of Health and Welfare of Korea, there is a possibility that the injury severity of pedestrian patients included was either biased or overestimated [5]. High-level emergency centers can accommodate patients transferred from lower-level hospitals, and the patients are more likely to be admitted from beginner to higherlevel hospitals through emergency medical services system of 119 field personnel. In addition, the results of this study could not be generalized to the entire population of South Korea. Moreover, comorbidities, including diabetes, coronary artery disease, and stroke, which are likely in elderly patients, were not accounted for due to missing data. Moreover, a lot of data for complications such as diabetes, coronary heart disease, and stroke were missing. The disease could be present in elderly patients involved in the study but was not reflected in the patient's medical status. Finally, because the data used in this study were based on EMR-ISS, there were limitations with respect to the use of the ISS, which is a conventionally used indicator in injured patients.

\section{CONCLUSION}

In this study, characteristics related to elderly pedestrian traffic accidents according to the severe and non-severe groups were identified using data from EDIIS. Compared with the non-severe group, the severe group included more women, older individuals, more ambulance users, and more hospitalizations; it was also associated with higher mortality. In addition, the results revealed that sex, age, use of ambulance, TA onset time, and types of vehicles were factors associated with the occurrence of injury severity. For injury severity to be reflected in the initial assessment and in the overall integrated treatment, it is recommended that physicians are informed of the characteristics of elderly pedestrian traffic accidents, and they 
should know the factors that affect the injury severity of elderly pedestrian patients. Furthermore, we believe that the study results should be reflected in the Road Traffic Act in South Korea to help in making relevant changes and developments to policies regarding elderly pedestrians.

\section{ACKNOWLEDGEMENTS}

This work was supported by clinical research grant from Pusan National University Hospital in 2019.

The authors are grateful to the Korea Center for Disease Control (KCDC) and Prevention for supporting data of Emergency Department-based Injury In-depth Surveillance (EDIIS).

\section{REFERENCES}

1. United Nations Population Division. World population aging, 1950-2050; 2002 [cited 2018 May 18]. Available from: https:// population.un.org/ProfilesOfAgeing2019/index.html.

2. Kim IK. Demographic transition and population aging in Korea. Korea J Popul Dev 1996;25:27-40.

3. OECD. OECD stat; 2018 [Internet]. Paris: OECD 2018 [cited 2018 May 18]. Available from: http://stats.oecd.org/.

4. Transportation Research Board. Special report 218: transportation in an aging society: improving mobility and safety for older persons. Vol. 1. Washington D.C.:National Research Council;1988.

5. Transportation Research Board. Driver and pedestrian training. In: McKnight AJ, Special report 218: transportation in an aging society: improving mobility and safety for older persons. Vol. 1. Washington D.C.:National Research Council;1988.

6. Traffic Accident Analysis System (TAAS). Elderly pedestrian accidents [Internet]. Wonju: TAAS 2019 [cited 2019 Jun 20]. Available from: http://taas.koroad.or.kr/sta/acs/gus/selectOdsnPdstrnTfcacd.do?menuId=WEB_KMP_OVT_MVT_TAS_PPT.
7. Mantilla J, Burtt D. Safer road design for older pedestrians [Internet]. Melbourne: Victoria Walks 2016 [cited 2019 Jun 20]. Available from: http://www.victoriawalks.org.au/Assets/Files/ Final_SRDOP_V1.1_low_res.pdf.

8. Ahn JY, Ryoo HW, Park JB, Kim JK, Lee MJ, Lee DE, et al. Comparison of traffic collision victims between older and younger drivers in South Korea: epidemiologic characteristics, risk factors and types of collisions. PLoS One 2019;14:e0214205.

9. Kim J, Shin SD, Im TH, Kug Jong Lee, Ko SB, Park JO, et al. Development and validation of the excess mortality ratio-adjusted injury severity score using the International classification of diseases 10th edition. Acad Emerg Med 2009;16:454-64.

10. Baker SP, O’Neill B. The injury severity score: an update. J Trauma 1976;16:882-5.

11. Stephenson S, Henley G, Harrison JE, Langley JD. Diagnosis based injury severity scaling: investigation of a method using Australian and New Zealand hospitalisations. Inj Prev 2004;10:379-83.

12. Dunbar G, Holland CA, Maylor EA. Older pedestrians: a critical review of the literature. London:Department for Transport;2004.

13. Clarke DD, Ward P, Bartle C, Truman W. Older drivers' road traffic crashes in the UK. Accid Anal Prev 2010;42:1018-24.

14. Lee JA, Choi H, Kim DA, Lee BS, Lee JJ, Bae JH, et al. Relationship between cognitive perceptual abilities and accident and penalty histories among elderly Korean drivers. Ann Rehabil Med 2016;40:1092-9.

15. Zegeer CV, Stutts JC, Huang H, Zhou M, Rodgman E. Analysis of elderly pedestrian accidents and recommended countermeasures. Transp Res Rec 1993;1405:56-63.

16. Sjögren H, Björnstig U, Eriksson A, Sonntag-Oström E, Oström M. Elderly in the traffic environment: analysis of fatal crashes in northern Sweden. Accid Anal Prev 1993;25:177-88.

17. Oström M, Eriksson A. Pedestrian fatalities and alcohol. Accid Anal Prev 2001;33:173-80.

18. Knoblauch RL, Crigler KL. Model pedestrian safety program user's guide. Virginia:Federal Highway Administration;1987.

19. Fred R. Walk alert: the new national pedestrian safety program. ITE Journal 1989;59:37-40. 